\title{
Characterization of Noncovalent Complexes of Antimalarial Agents of the Artemisinin-Type and FE(III)-Heme by Electrospray Mass Spectrometry and Collisional Activation Tandem Mass Spectrometry
}

\author{
Vlada A. Pashynska,* Hilde Van den Heuvel, and Magda Claeys \\ Department of Pharmaceutical Sciences, University of Antwerp, Antwerp, Belgium \\ Marina V. Kosevich \\ Verkin Institute for Low Temperature Physics and Engineering, National Academy of Sciences of Ukraine, \\ Kharkov, Ukraine
}

\begin{abstract}
In this study, we demonstrate, using electrospray ionization mass spectrometry (ESI-MS) and collision-induced dissociation tandem mass spectrometry (ESI-MS/CID/MS), that stable noncovalent complexes can be formed between Fe(III)-heme and antimalarial agents, i.e., quinine, artemisinin, and the artemisinin derivatives, dihydroartemisinin, $\alpha$ - and $\beta$-artemether, and $\beta$-arteether. Differences in the binding behavior of the examined drugs with $\mathrm{Fe}(\mathrm{III})$-heme and the stability of the drug-heme complexes are demonstrated. The results show that all tested antimalarial agents form a drug-heme complex with a 1:1 stoichiometry but that quinine also results in a second complex with the heme dimer. ESI-MS performed on mixtures of pairs of various antimalarial agents with heme indicate that quinine binds preferentially to $\mathrm{Fe}(\mathrm{III})$-heme, while ESI-MS/CID/MS shows that the quinine-heme complex is nearly two times more stable than the complexes formed between heme and artemisinin or its derivatives. Moreover, it is found that dihydroartemisinin, the active metabolite of the artemisinin-type drugs in vivo, results in a $\mathrm{Na}^{+}$-containing heme-drug complex, which is as stable as the heme-quinine complex. The efficiency of drug-heme binding of artemisinin derivatives is generally lower and the decomposition under CID higher compared with quinine, but these parameters are within the same order of magnitude. These results suggest that the efficiency of antimalarial agents of the artemisinin-type to form noncovalent complexes with Fe(III)heme is comparable with that of the traditional antimalarial agent, quinine. Our study illustrates that electrospray ionization mass spectrometry and collision-induced dissociation tandem mass spectrometry are suitable tools to probe noncovalent interactions between heme and antimalarial agents. The results obtained provide insights into the underlying molecular modes of action of the traditional antimalarial agent quinine and of the antimalarials of the artemisinin-type which are currently used to treat severe or multidrug-resistant malaria. (J Am Soc Mass Spectrom 2004, 15, 1181-1190) (c) 2004 American Society for Mass Spectrometry
\end{abstract}

$\mathrm{T}$ The major problem in the treatment of malaria is the increasing resistance of the malarial parasites, e.g., Plasmodium falciparum, to the commonly used antimalarial drugs, i.e., quinolines [1-3]. The quinolines, particularly quinine, chloroquine, and mefloquine, have been the basic drugs for treatment of malaria during the past 50 years and are still widely

Published online June 19, 2004

Address reprint requests to Dr. M. M. Claeys, Department of Pharmaceutical Sciences, University of Antwerp, Universiteitsplein 1, B-2610 Antwerp, Belgium. E-mail: magda.claeys@ua.ac.be

${ }^{*}$ On leave from Verkin Institute for Low Temperature Physics and Engineering, National Academy of Sciences of Ukraine, Kharkov 61103, Ukraine. used in clinical practice. The antimalarial effect of the quinolines is due to inhibition of the polymerization pathway of free heme, which is released inside the food vacuole of the parasite after hemoglobin proteolysis and is toxic to the parasite owing to its oxidative damage to cell membranes and other biomolecules [4]. It has been demonstrated that the underlying molecular mechanism of the quinolines is based on association of the drugs with $\mathrm{Fe}(\mathrm{III})$-heme and heme polymer, i.e., noncovalent binding, which can interfere with the heme polymerization pathway [5-7]. The polymerized heme accumulates in the form of an insoluble, microcrystalline black-brown pigment called hemozoin or the ma- 

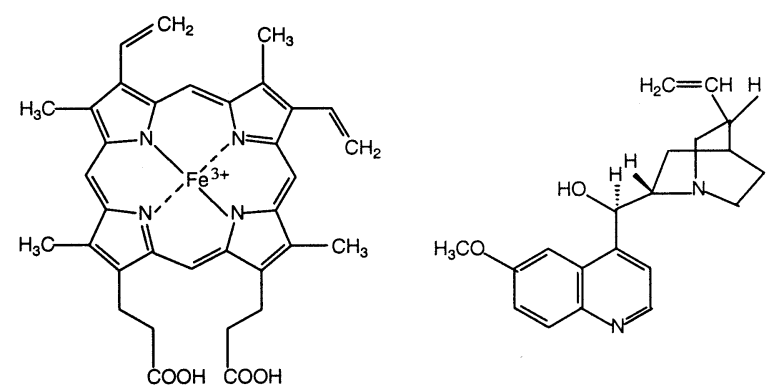

$\mathrm{Fe}(\mathrm{III})$-heme [Fe(III)protoporphyrin IX]

Quinine

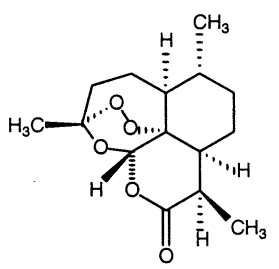

Artemisinin

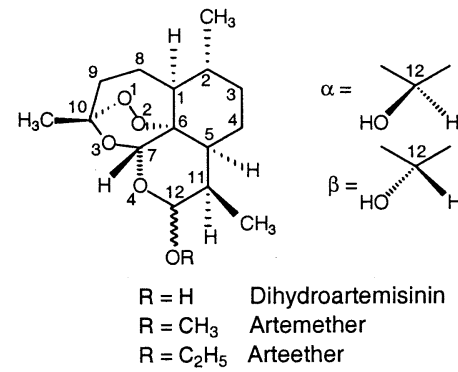

$\mathrm{R}=\mathrm{C}_{2} \mathrm{H}_{5}$ Arteether

Scheme 1. Structures of Fe(III)-heme, quinine and the artemisinin-type drugs examined in the present study.

laria pigment, which is identical to synthetic $\beta$-hematin and is not a polymer as previously thought but a repeating array of coordinated dimers, bound through reciprocal iron(III)-carboxylate bonds to one of the propionic side chains of each porphyrin and held together in a crystalline matrix by hydrogen bonding interactions [8]. Because the polymerization pathway is unique to the malarial parasite, it offers an attractive target for the design of new antimalarials.

The first antimalarial compound to be discovered, which also served as the lead compound for synthetic antimalarials of the quinoline-type, was the alkaloid quinine isolated from Chinchona bark (for a historical account, see [9]). Many other leads for potential new antimalarials have been characterized since then, and several of them have been isolated from medicinal plants. One of these plants is Artemisia annua, which has been used in Chinese traditional medicine (Quinghaosu) for more than two thousand years as a herbal anti-fever remedy [1, 10]. In 1969, a promising lead compound, artemisinin, was isolated from Artemisia annua by the Chinese scientist Zhenxing Wei [10], and since then a number of artemisinin derivatives have been synthesized and tested. Artemisinin and its derivatives are currently successfully used to treat severe or multidrug-resistant Plasmodium falciparum malaria, including cerebral malaria [1, 11]. However, the exact molecular mechanisms of drugs of the artemisinin-type are still not properly understood and are, therefore, a current topic of active investigation [12]. Artemisinin is a sesquiterpene lactone bearing an endoperoxide function (Scheme 1), which was shown to be crucial for antimalarial activity. The heme-catalyzed cleavage of the endoperoxide bridge of artemisinin and formation of toxic free radicals have received considerable attention as a likely molecular mechanism for the antimalarial activity of artemisinin and its derivatives [13]. Formation of non-polymerizable alkylated heme derivatives has been demonstrated in vitro and evidence has been provided that $\mathrm{C}$-centered radicals derived from artemisinin act as alkylating agents [14, 15]. Furthermore, it has been shown that covalent artemisinin-heme adducts mimick heme in binding to the enzyme histidine-rich protein II and as such have the ability to inhibit heme polymerization [16]. Recently, evidence has been obtained that iron-activated artemisinin-type drugs act by alkylating an essential enzyme of Plasmodium falciparum, i.e., a sarco/endoplasmic reticulum $\mathrm{Ca}^{2+}$-ATPase ortholog [17].

Formation of a relatively stable noncovalent complex between antimalarial drugs of the artemisinin-type and $\mathrm{Fe}$ (II)-heme can be regarded as the first step in covalent interaction, since $\mathrm{Fe}^{2+}$ is required for activation of artemisinin derivatives, i.e., generation of alkyl radicals $[17,18]$. Experimental evidence using spectrophotometry has demonstrated that noncovalent interaction indeed occurs prior to covalent association in in vitro experiments [19]. A theoretical model based on a combination of molecular docking and a three-dimensional quantitative structure-activity study, developed by Cheng et al. [20], revealed a good correlation between antimalarial activity and the binding energy of artemisinin derivatives with Fe(II)-heme. The global energyminimum configuration of the artemisinin type drugheme complex indicates that the endoperoxide bridge of the drug molecule exactly points towards the Fe ion of heme and the two carboxyl ethyl groups in heme are perpendicular to the porphyrin ring, their carboxyl groups interacting with the hydrogens attached to the C1 and C9 carbons (Scheme 1) of the artemisinin derivatives (Figure 3 in [20]). The latter study showed that the electrostatic energy comprises about two thirds of the total binding energy, which includes also steric and hydrophobic components. Since the electrostatic component revealed the same trend of variation (increase or decrease) as the total stabilization energy with variation of the structure of the artemisinin-type drugs, it was suggested that the electrostatic component alone permits evaluation of the stability of the complexes.

The present study deals with the characterization of noncovalent complexes formed in vitro between Fe(III)heme and artemisinin-type drugs as a model system for the interaction between the latter drugs and Fe(III)heme, $\mathrm{Fe}(\mathrm{II})$-heme or $\mathrm{Fe}(\mathrm{II})$-heme-containing proteins, which may occur in vivo. In order to evaluate the potential of the drugs to form noncovalent complexes with $\mathrm{Fe}(\mathrm{III})$-heme and their relative binding strengths, we resorted to electrospray ionization mass spectrometry (ESI-MS) and collision-induced dissociation tandem mass spectrometry (ESI-MS/CID/MS). Electrospray ionization mass spectrometry provides a rapid, sensitive and highly selective tool for probing noncova- 
lent interactions [21-24]. Most studies that are based on this approach rely on the ability of ESI to transfer noncovalent solution-phase assemblies intact into the gas phase. The validity of this strategy has been supported in numerous studies, especially for experiments on protein-protein and protein-ligand interactions (for a review, see [25]), including studies on heme-containing proteins [26]. The relative binding strengths between the drugs and Fe(III)-heme was assessed using lowenergy collision-induced dissociation. This approach has previously been shown to be useful in determining the structure-activity relationship of antimalarial agents, namely, terpene isonitriles [27] and neocryptolepine derivatives [28]. We demonstrate in the present work that artemisinin-type drugs form a noncovalent complex with Fe(III)-heme in vitro, which is weaker than that with quinine. However, in contrast to quinine a complex with the dimeric form of Fe(III)-heme or $\beta$-hematin could not be detected. Dihydroartemisinin, the active metabolite of artemisinin derivatives in vivo [1], is shown to form a second more stable complex in which a hydrogen is replaced by a sodium atom, which has a binding strength comparable with that of the quinine-heme complex.

\section{Experimental}

\section{Materials}

Hemin (i.e., Fe(III)protoporphyrin IX chloride, purity $>98 \%$ ) was obtained from Fluka (Buchs, Switzerland), and quinine sulfate salt (purity, 90\%) was purchased from Sigma (St. Louis, MO). Artemisinin and its derivatives, dihydroartemisinin, $\alpha$ - and $\beta$-artemether, and $\beta$-arteether, were a gift from Dafra Pharma (OudTurnhout, Belgium). The tested artemisinin-type drugs had a purity $>99 \%$. The structures of Fe(III)-heme (hereafter, referred to as heme), quinine and the artemisinin-type drugs investigated in this study are presented in Scheme 1. Methanol (super grade) was purchased from Lab-Scan (Dublin, Ireland).

Abbreviations: $\mathrm{FP}^{+}$, heme or $\mathrm{Fe}(\mathrm{III})$ protoporphyrin IX (cationic mass, $616 \mathrm{Da}$ ); An, artemisinin (MW 282); DHAn, dihydroartemisinin (MW 284); $\alpha$ - and $\beta$-Am, $\alpha$ and $\beta$-isomers of artemether, respectively (MW 298); Ae, $\beta$-arteether (MW 312); Qn, quinine (MW 324).

\section{Sample Preparation}

Stock solutions of heme were prepared using hemin and contained $5 \mathrm{mM}$ in $\mathrm{MeOH}$ (plus a drop of $\mathrm{NH}_{4} \mathrm{OH}$ to solubilize heme), while those of quinine, artemisinin, and its derivatives, dihydroartemisinin, artemether, and arteether, contained $5 \mathrm{mM}$ of the drug in $\mathrm{MeOH}$ : $\mathrm{CH}_{2} \mathrm{Cl}_{2}$ (19:1; vol/vol). Mixtures of the drugs with heme were prepared in $\mathrm{MeOH}$ with different drug: heme concentration ratios starting from the stock solutions. In the final solutions, water was added in order to ensure the stabilization of the ESI process. The final $\mathrm{pH}$ of the solutions was about 7 , at which the iron atom in the heme molecule is known to be in the Fe(III) state [27]. Drug-heme mixtures with a molar concentration ratio $1: 1$ contained $250 \mu \mathrm{M}$ drug and $250 \mu \mathrm{M}$ heme in MeOH: $\mathrm{H}_{2} \mathrm{O}$ (3:1; vol/vol); for the drug-heme mixtures with a concentration ratio 1:2 the concentration of the drug was $125 \mu \mathrm{M}$, while that of heme was $250 \mu \mathrm{M}$ in MeOH: $\mathrm{H}_{2} \mathrm{O}$ (3:1; vol/vol). For the three-component mixtures (drug 1/drug 2/heme), mixtures were prepared with several concentration molar ratios, i.e., 1:1:1 (125 $\mu \mathrm{M}$ of drug 1, $125 \mu \mathrm{M}$ of drug 2, $125 \mu \mathrm{M}$ heme in MeOH: $\mathrm{H}_{2} \mathrm{O}$ (3:1; vol/vol)), 1:1:2 (125 $\mu \mathrm{M}$ of drug 1, 125 $\mu \mathrm{M}$ of drug $2250 \mu \mathrm{M}$ heme in $\mathrm{MeOH}: \mathrm{H}_{2} \mathrm{O}$ (3:1; vol/ vol)) and 1:1:4 (125 $\mu \mathrm{M}$ of drug 1, $125 \mu \mathrm{M}$ of drug 2500 $\mu \mathrm{M}$ heme in $\mathrm{MeOH}: \mathrm{H}_{2} \mathrm{O}(3: 1$; vol/vol)). The final mixtures were subjected to electrospray ionization mass spectrometry or to collision-induced dissociation tandem mass spectrometry.

\section{Mass Spectrometry}

The mass spectral data were obtained in the positive ion mode on an Autospec-oa-TOF mass spectrometer (Micromass, Manchester, UK), which was equipped with an electrospray ionization source. The electrospray ionization source was operated at $4 \mathrm{kV}$, while the electrospray needle was operated at a maximum voltage of 3 $\mathrm{kV}$ (i.e., relative to the pepper pot counter electrode which is at $5 \mathrm{kV}$ ). Nitrogen was used both as bath gas $\left(100{ }^{\circ} \mathrm{C} ; 250 \mathrm{~L} / \mathrm{h}\right)$ and as nebulizing gas $(15 \mathrm{~L} / \mathrm{h})$. First-order ESI mass spectra were recorded in the mass range $m / z \quad 100-1600$. The drug-heme solutions were introduced into the mass spectrometer by a syringe pump (Harvard Apparatus, South Natick, MA), employing a $100 \mu \mathrm{L}$ syringe, at a constant flow rate of 5 $\mu \mathrm{L} / \mathrm{min}$. The effect of the cone voltage on the intensity of the signal of the drug-heme complexes was examined. Low- and high-energy collision-induced dissociation (CID) spectra were acquired at a collision energy $\left(\mathrm{E}_{\text {lab }}\right)$ of $400 \mathrm{eV}$ using $\mathrm{He}$ and $\mathrm{Xe}$, respectively, as collision gas. Helium or xenon was introduced into the collision cell until the signal of the very weak [FP: $\left.\mathrm{CH}_{3} \mathrm{OH}\right]^{+}$complex $(\mathrm{m} / \mathrm{z} 648)$ reached about $80 \%$ of its original value. In order to ensure reproducible results the setting of the collision cell gas pressure was not changed during our experiments. Data acquisition and processing were performed using OPUS V3.1X software. All scans were acquired in the continuum mode.

\section{Results and Discussion}

\section{Optimization of ESI Conditions for Detection of Noncovalent Complexes Between Artemisinin-Type Drugs and Heme}

In a first series of experiments, we recorded first-order ESI mass spectra of heme, the drugs and binary drugheme mixtures and optimized the ESI conditions. In particular, we noted that the potential on the first 


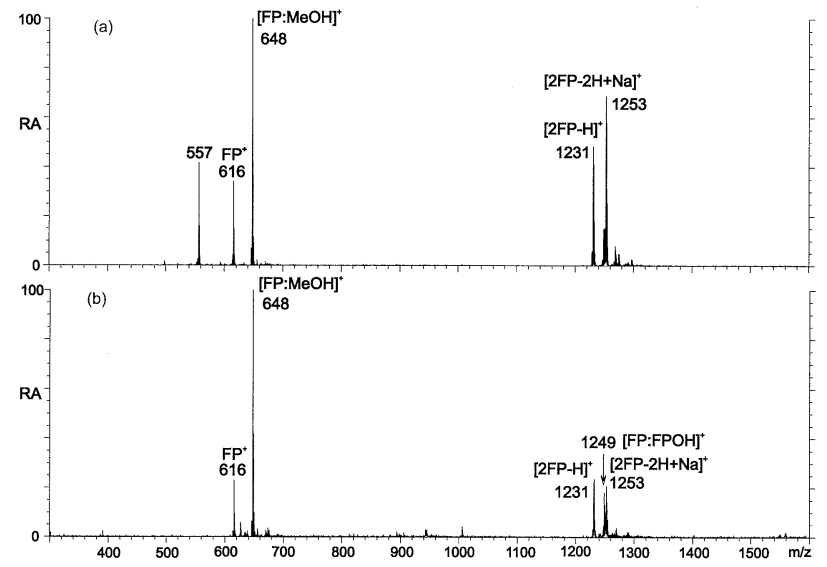

Figure 1. ESI mass spectra obtained for $\mathrm{Fe}(\mathrm{III})$-heme in $\mathrm{MeOH}$ : $\mathrm{H}_{2} \mathrm{O}(3: 1, \mathrm{vol} / \mathrm{vol})$ at (a) non-optimal cone voltage, $150 \mathrm{~V}$, and (b) optimal cone voltage, $75 \mathrm{~V}$. The needle voltage was $2.3 \mathrm{kV}$.

skimmer (cone voltage) had to be adjusted in order to reduce in-source fragmentation of heme $(\mathrm{m} / \mathrm{z}$ 616), which was evident from the formation of an ion at $\mathrm{m} / \mathrm{z}$ 557 due to loss of the ethylcarboxyl side chain (Figure 1). The loss of a $\mathrm{CH}_{2}-\mathrm{COOH}$ radical $(59 \mathrm{u})$ is consistent with the structure of Fe(III)-heme, shown in Scheme 1. The same procedure was performed for the binary drug-heme mixtures. Gradual variation of the cone voltage allowed the establishment of an optimal value of $75 \mathrm{~V}$ at which the signals of the drug-heme complexes were the highest and fragmentation was the lowest. Optimization of the needle voltage was also performed because it was found that the dihydroartemisinin-heme complex is susceptible to reduction. Oxidation-reduction processes are known to occur during electrospray ionization and have been well documented (for a review, see [29]). At high needle voltage ( $>2.5$ $\mathrm{kV})$, the isotopic cluster of the dihydroartemisininheme complex ([DHAn:FP] ${ }^{+} \mathrm{m} / \mathrm{z}$ 900) was severely distorted by a hydrogenated species, which corresponds to [DHAn:FP $+\mathrm{H}^{+}{ }^{+}(\mathrm{m} / \mathrm{z}$ 901) containing protonated $\mathrm{Fe}(\mathrm{II})$-heme. It is worth mentioning that protonated $\mathrm{Fe}(\mathrm{II})$-heme $(\mathrm{m} / \mathrm{z} 617)$ has also been reported for electrospray ionization of the complex between heme and cytochrome $c$ [26]. Gradual variation of the needle voltage, employing a dihydroartemisinin-quinine-heme mixture (1:1:1), allowed the establishment of an optimal value of $2.3 \mathrm{kV}$ for the signal intensity of the dihydroartemisinin-heme complex without affecting the isotopic pattern as well as for the signal intensity of the quinine-heme complex.

Using these optimized conditions, first-order ESI mass spectra were obtained for drug-heme binary mixtures (1:2). In order to allow the detailed interpretation of the spectra, we also recorded the spectrum of heme. Figure 1a and $b$ show the ESI spectra obtained for heme under non-optimal and optimal conditions, respectively. Under optimal conditions, the following ions can be noted in the spectrum of heme: $\mathrm{FP}^{+}(m / z$ 616), $[\mathrm{FP}: \mathrm{MeOH}]^{+}\left(m / z\right.$ 648), $[2 \mathrm{FP}-\mathrm{H}]^{+}(m / z$ 1231), [FP:

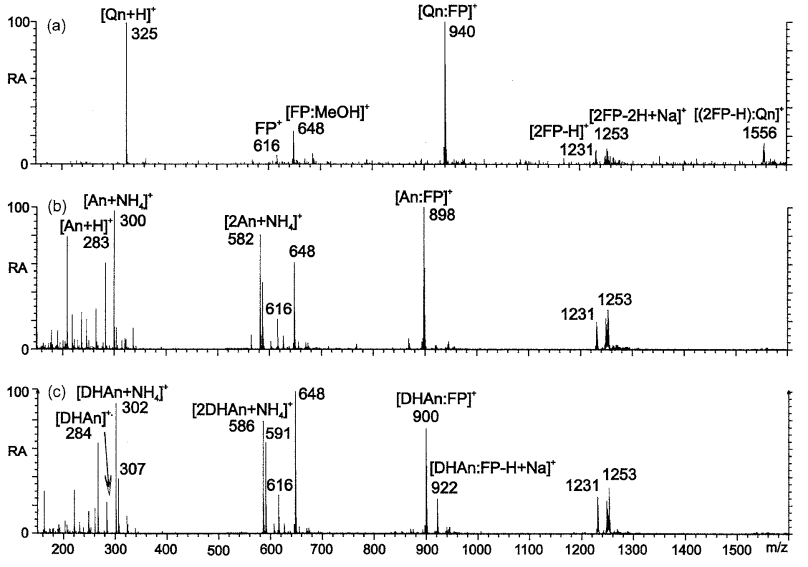

Figure 2. ESI mass spectra of two-component mixtures: (a) quinine-heme (1:2), (b) artemisinin-heme (1:2), and (c) dihydroartemisinin-heme (1:2).

$\mathrm{FPOH}^{+}\left(m / z\right.$ 1249) and $[2 \mathrm{FP}-2 \mathrm{H}+\mathrm{Na}]^{+}(m / z 1253)$. The formation of heme dimer-related ions (i.e., [2FP $\mathrm{H}]^{+}$and $[2 \mathrm{FP}-2 \mathrm{H}+\mathrm{Na}]^{+}$) is worth noting. Their basic structures likely correspond to that of the dimer units of $\beta$-hematin or hemozoin. The ion at $m / z 1249$, corresponding to [FP:FPOH $]^{+}$, can be regarded as a noncovalent complex between heme and hematin (i.e., $\mathrm{Fe}(\mathrm{III})$ protoporphyrin IX hydroxide).

Quinine-heme mixture. Before examining the artemisinin-type drugs, we first examined quinine which is known to bind to heme [5-7]. Figure 2a illustrates the ESI mass spectrum obtained for the quinine-heme (1:2) mixture. Formation of a quinine-heme noncovalent complex is evident from the intense peak [Qn:FP] ${ }^{+}(\mathrm{m} / \mathrm{z}$ 940, RA 97\%). In addition, a complex of quinine with the heme dimer [(2FP - H):Qn] ${ }^{+}(m / z$ 1556, RA 10\%) can be noted. Formation of a noncovalent complex with heme dimer or $\beta$-hematin has been detected previously for neocryptolepine [28]. Peaks related to heme, namely, $[\mathrm{FP}]^{+}\left(m / z\right.$ 616, RA 6\%), [FP:MeOH] ${ }^{+}(m / z$ 648, RA $18 \%),[2 \mathrm{FP}-\mathrm{H}]^{+}(\mathrm{m} / \mathrm{z} 1231, \mathrm{RA} 7 \%),[2 \mathrm{FP}-2 \mathrm{H}+$ $\mathrm{Na}^{+}(\mathrm{m} / z$ 1253, RA $11 \%)$, and quinine [QnH] ${ }^{+}(\mathrm{m} / z$ 325, RA $100 \%$ ) are also present.

Artemisinin-heme mixture. The ESI mass spectrum obtained for the artemisinin-heme (1:2) mixture is shown in Figure $2 \mathrm{~b}$. The artemisinin-heme noncovalent complex [An:FP] ${ }^{+}(m / z$ 898) is the most abundant ion in the spectrum. It is interesting to note that the artemisininheme complex is stable upon electrospray ionization. One could expect that artemisinin which contains a labile endoperoxide bridge would give rise to radical formation in electrospray ionization, but apparently this is not the case. In contrast to quinine, a complex of artemisinin with the heme dimer could not be detected. The other peaks in the spectrum could easily be attributed to ions characteristic of the individual components of the mixture, i.e., $[\mathrm{An}+\mathrm{H}]^{+}(\mathrm{m} / \mathrm{z} 283$, RA 61\%), [An $\left.+\mathrm{NH}_{4}\right]^{+}(m / z 300, \mathrm{RA} 98 \%),\left[2 \mathrm{An}+\mathrm{NH}_{4}\right]^{+}(m / z 582$, 
Table 1. Major peaks in the ESI mass spectrum of a dihydroartemisinin-heme (1:2) mixture

\begin{tabular}{|c|c|c|}
\hline$m / z$ & Peak identification & RA (\%) \\
\hline 284 & [DHAn] $^{+\cdot}$ & 22 \\
\hline 302 & {$\left[\mathrm{DHAn}+\mathrm{NH}_{4}\right]^{+}$} & 92 \\
\hline 307 & {$[\mathrm{DHAn}+\mathrm{Na}]^{+}$} & 38 \\
\hline 586 & {$\left[2 \mathrm{DHAn}+\mathrm{NH}_{4}\right]^{+}$} & 80 \\
\hline 591 & {$[2 \mathrm{DHAn}+\mathrm{Na}]^{+}$} & 65 \\
\hline 616 & {$[\mathrm{FP}]^{+}$} & 28 \\
\hline 648 & ${ }^{[\mathrm{FP}: \mathrm{MeOH}]^{+}}$ & 100 \\
\hline 900 & [DHAn:FP] $^{+}$ & 75 \\
\hline 922 & {$[\mathrm{DHAn:FP}-\mathrm{H}+\mathrm{Na}]^{+}$} & 25 \\
\hline 1231 & $[2 \mathrm{~F}]-\mathrm{H}]^{+}$ & 28 \\
\hline 1253 & {$[2 \mathrm{FP}-2 \mathrm{H}+\mathrm{Na}]^{+}$} & 33 \\
\hline
\end{tabular}

RA 80\%), $\mathrm{FP}^{+}\left(\mathrm{m} / \mathrm{z}\right.$ 616, RA 22\%), [FP:MeOH] ${ }^{+}(\mathrm{m} / \mathrm{z} 648$, RA 62\%), [2FP - H] $]^{+}(m / z) 1231$, RA 20\%), [2FP - 2H + $\mathrm{Na}^{+}(\mathrm{m} / \mathrm{z}$ 1253, RA $30 \%)$. These data provide experimental evidence that artemisinin just as quinine can form a noncovalent complex with heme in solution. It is worth noting that the solution consisted of $\mathrm{MeOH}: \mathrm{H}_{2} \mathrm{O}$ in a molar ratio of 3:1 and that even in the presence of a relatively high concentration of the organic solvent methanol an intense peak for the drug-heme complex could be generated, consistent with the strong electrostatic nature of the drug-heme complex. However, it is pointed out that there is a distinct difference between quinine and artemisinin in that quinine also forms a noncovalent complex with dimeric heme, which is relevant to its underlying molecular mechanism of action. It has namely been suggested that quinolinetype drugs such as chloroquinine and quinidine interact with both the heme monomer and dimer [6], which is in agreement with our mass spectrometric observations.

Dihydroartemisinin-heme mixture. Dihydroartemisinin is of particular interest because it is the active metabolite of semi-synthetic derivatives of artemisinin in vivo $[1,30]$. The major peaks of the ESI mass spectrum obtained for the binary mixture of dihydroartemisinin with heme (1:2) (Figure 2c) are summarized in Table 1. The formation of a radicalar molecular ion species for dihydroartemisinin (i.e. [DHAn] ${ }^{+*}, \mathrm{~m} / z$ 284) is worth noting. This [DHAn] ${ }^{+\cdot}$ ion can be explained by electrochemical oxidation in the electrospray needle [29]. Taking into account that molecular radical formation is not observed for artemisinin, artemether and arteether, it can be suggested that an intact 12-hydroxyl group facilitates radical formation.

The data obtained for dihydroartemisinin (Figure 2c and Table 1) demonstrate that dihydroartemisinin just as artemisinin forms a noncovalent complex with heme [DHAn:FP] ${ }^{+}(m / z$ 900). The formation of a noncovalent dihydroartemisinin-heme complex with 1:1 stoichiometry is in agreement with the study by Messori et al. [19] which used spectrophotometric determinations. In addition, dihydroartemisinin also results in a complex with heme in which a hydrogen is replaced by a sodium
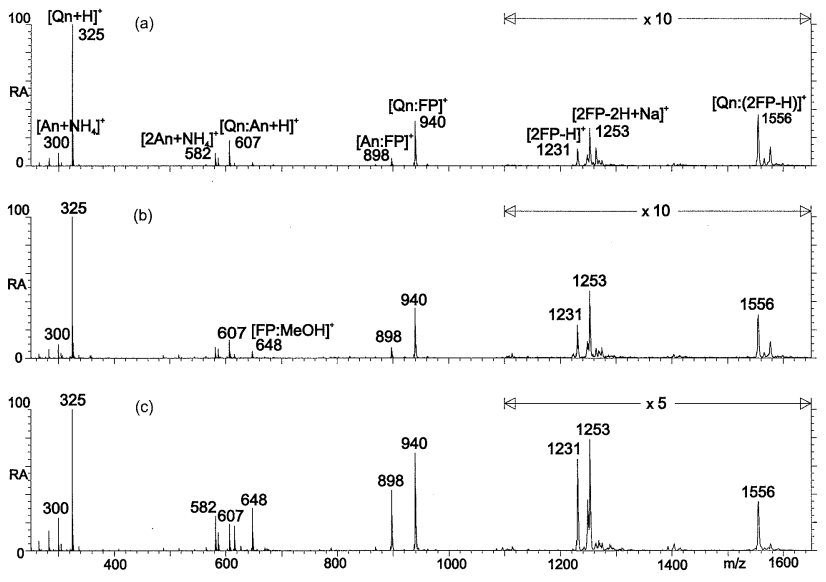

Figure 3. ESI mass spectra of three-component quinine-artemisinin-heme mixtures with molar ratios of (a) 1:1:1, (b) 1:1:2, and (c) 1:1:4.

atom [DHAn:FP $-\mathrm{H}+\mathrm{Na}]^{+}(\mathrm{m} / \mathrm{z}$ 922), which was not detected for either quinine or artemisinin. As sodium ions are ubiquitous in physiological conditions, it is logical to assume that this complex may be relevant to in vivo conditions.

\section{Estimation of the Relative Efficiency of Drugs to Form Drug-Heme Complexes}

For estimation of the relative efficiency of the drugs to form noncovalent complexes with heme, we examined three-component mixtures containing a reference drug, the drug to be tested, and heme. In these mixtures two drugs, the drug to be tested and the reference drug, competed for heme. Two drugs were selected as reference drugs, namely, quinine as a well-known and highly efficient antimalarial drug and artemisinin as the lead compound of the artemisinin-type series.

Quinine-artemisinin-hememixture. Quinine-artemisininheme three-component mixtures in different molar concentration ratio, 1:1:1, 1:1:2 and 1:1:4, were tested (Figure 3).

In the ESI mass spectra obtained for all these mixtures, peaks related to the individual components were present, i.e., $[\mathrm{Qn}+\mathrm{H}]^{+}(m / z 325),[\mathrm{An}+\mathrm{H}]^{+}(m / z 283)$, $\left[\mathrm{An}+\mathrm{NH}_{4}\right]^{+}\left(m / z\right.$ 300), $\left[2 \mathrm{An}+\mathrm{NH}_{4}\right]^{+}(\mathrm{m} / z$ 582), $[\mathrm{FP}: \mathrm{MeOH}]^{+}(m / z 648)$, and $\left[2 \mathrm{FP}-2 \mathrm{H}+\mathrm{Na}^{+}(\mathrm{m} / \mathrm{z}\right.$ 1253). For the three-component mixtures, the same types of noncovalent drug-heme complexes were detected as in the experiments with binary mixtures, i.e., [Qn:FP] $^{+}\left(m / z\right.$ 940), [(2FP - H):Qn] ${ }^{+}(m / z$ 1556) and [An:FP] $^{+}(m / z$ 898).

The general analysis of the spectra allows us to suggest that quinine binds more efficiently with heme in comparison with artemisinin, since the abundance of the quinine-heme complex is higher than the artemisinin-heme complex for all molar ratios tested. At the same time, certain concentration dependences were 

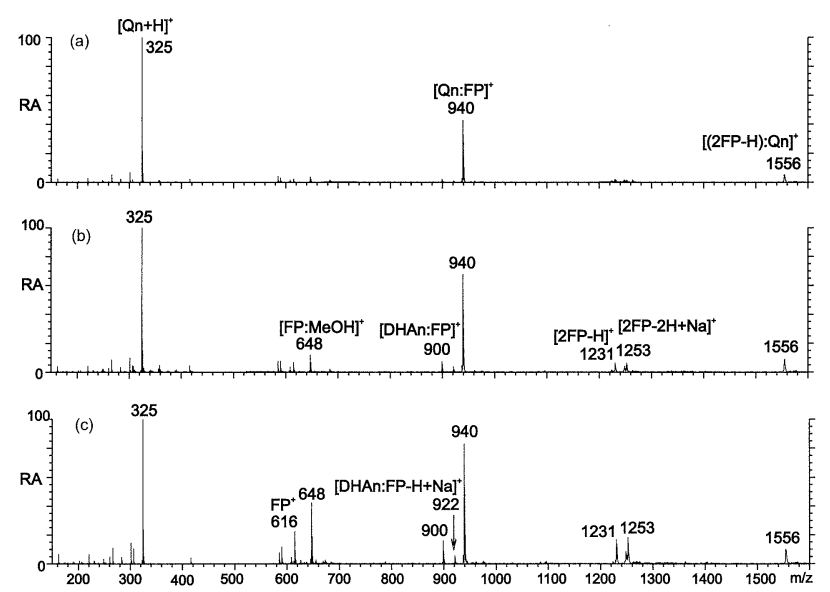

Figure 4. ESI mass spectra of three-component quinine-dihydroartemisinin-heme mixtures with molar ratios of (a) 1:1:1, (b) 1:1:2, and (c) 1:1:4.

observed. The relative abundances (RAs) of the drugheme noncovalent complexes increased with the heme concentration, for the $[\mathrm{Qn}: \mathrm{FP}]^{+}$complex, starting at a RA of $32 \%$ and increasing to 40 and $70 \%$ ( Figure $3 a, b$, c), and, for the $[\mathrm{An}: \mathrm{FP}]^{+}$complex, starting at a RA of $6 \%$ and increasing to 10 and $40 \%$. Along with this, the relative abundance of the $[\mathrm{An}: \mathrm{FP}]^{+}$complex in relation to that of the [Qn:FP] $]^{+}$complex increased from 19 to 25 and $57 \%$ with an increase of the heme concentration in the mixtures. These results indicate that there is a competition between the two drugs for binding with heme in solution: under the conditions of a heme deficit, quinine binds preferentially, while at a heme molar excess more intense binding of artemisinin becomes possible. The efficiency of artemisinin to bind noncovalently with heme, however, remains lower than that of quinine even at a four-fold molar excess of heme in relation to both drugs.

Quinine-dihydroartemisinin-heme mixture. The same experiment as described above was performed with quinine-dihydroartemisinin-heme mixtures (Figure 4). Noncovalent drug-heme complexes were detected for $[\mathrm{Qn}: \mathrm{FP}]^{+}(m / z$ 940) with a relatively high abundance and for $[(2 \mathrm{FP}-\mathrm{H}): \mathrm{Qn}]^{+}\left(m / z\right.$ 1556) and [DHAn:FP] ${ }^{+}$ $(\mathrm{m} / \mathrm{z}$ 900) with a low RA. With the growth of the concentration of heme in the mixtures (molar ratios 1:1:2 and 1:1:4), the relative intensities of complex peaks also increased. For the 1:1:2 mixture (Figure $4 \mathrm{~b}$ ) the ions, corresponding to the quinine complexes with heme, [Qn:FP] ${ }^{+}\left(m / z\right.$ 940) and [(2FP - H):Qn] ${ }^{+}(m / z$ 1556), had a RA of 68 and $11 \%$, respectively, while the RA of [DHAn:FP] ${ }^{+}(\mathrm{m} / \mathrm{z} 900)$ increased to $8 \%$. A peak of the sodium-containing complex [DHAn:FP $-\mathrm{H}+\mathrm{Na}]^{+}$ $(\mathrm{m} / \mathrm{z}$ 922), discussed above for the binary DHAn-heme system (see Figure 2c), appears here with a RA of 3\%. The results obtained for the 1:1:4 mixture (Figure 4c) show that the intensity of the DHAn complexes with heme further increased, i.e., [DHAn:FP] ${ }^{+}(m / z$ 900, RA 17\%) and [DHAn:FP $-\mathrm{H}+\mathrm{Na}^{+}(\mathrm{m} / \mathrm{z}$ 922, RA 5\%).
Table 2. Relative abundances of drug-heme complexes in relation to artemisinin-heme observed in the ESI mass spectra of three-component mixtures (1:1:2)

\begin{tabular}{|c|c|c|}
\hline $\begin{array}{l}\text { Noncovalent complex } \\
\text { drug:heme }\end{array}$ & $m / z$ & $\begin{array}{l}\text { TA (\%) } \\
(\mathrm{N}=3)\end{array}$ \\
\hline$[\mathrm{An}: \mathrm{FP}]^{+}$ & 898 & 100 \\
\hline$[\mathrm{DHAn:FP}]^{+}$ & 900 & $63 \pm 1.5 \%$ \\
\hline$[\mathrm{DHAn:F}]-\mathrm{H}+\mathrm{Na}]^{+}$ & 922 & $29 \pm 2.5 \%$ \\
\hline$[\alpha-\mathrm{Am}: \mathrm{FP}]^{+}$ & 914 & $36 \pm 2.6 \%$ \\
\hline$[\beta-\mathrm{Am}: \mathrm{FP}]^{+}$ & 914 & $22 \pm 1.3 \%$ \\
\hline$[\mathrm{AE}: \mathrm{FP}]^{+}$ & 928 & $22 \pm 3.5 \%$ \\
\hline
\end{tabular}

The results obtained for the quinine-dihydroartemisinin-heme mixtures demonstrate that quinine is the most efficient in forming a noncovalent complex with heme in equimolar mixtures but that with increase of the heme concentration dihydroartemisinin-heme complex formation also increases. From the comparison of the results obtained for the three-component mixtures discussed above, it follows that artemisinin is slightly more effective than dihydroartemisinin in binding with heme. In subsequent experiments aimed at evaluating more in detail the relative efficiency of the various artemisinin derivatives, we have, therefore, selected artemisinin as a reference compound.

Artemisinin-dihydroartemesinin-heme, artemisinin- $\beta$-arteether-heme, artemisinin- $\alpha$-artemether-heme and artemisinin$\beta$-artemether-heme mixtures. Mixtures with a molar ratio of 1:1:2 were examined, and for all the threecomponent mixtures tested, drug-heme complexes were detected with the artemisinin-heme complex $(\mathrm{m} / \mathrm{z}$ 898) dominating the spectrum. In Table 2 the comparison is shown of the RAs of the drug-heme complexes in relation to that of artemisinin-heme.

The data obtained for the three-component mixtures containing artemisinin as a reference compound suggest that artemisinin is favored compared with its derivatives for complex formation with heme. Dihydroartemisinin, the active metabolite formed from artemisinin derivatives in vivo, is distinguished in the set of the artemisinin derivatives in that it is the only compound which forms two types of complexes with heme, i.e., the expected one ([DHAn:FP] $]^{+}$) and the corresponding Na-containing complex (see Table 2). Similar results were obtained for the three-component mixtures containing quinine as a reference compound, discussed above. Furthermore, our data reveal that the stereoisomers of artemether have a different efficiency to form a noncovalent complex with heme, the $\alpha$-isomer being about a 1.5 times more efficient than the $\beta$-isomer. This experimental observation is in agreement with that derived by a theoretical model based on a combination of molecular docking and a three-dimensional quantitative structure-activity study [20], which illustrates that $\alpha$-isomers of artemisinin derivatives are, in general, more active than the corresponding $\beta$-isomers.

Although the ESI technique is widely used in studies 
of noncovalent complexes of biomolecules [21-26], the correspondence between the specific noncovalent complexes formed in solution and gas-phase cluster ions produced by the ESI technique may be questioned [31-33]. Concern has been expressed that single molecules present in the solute can be randomly distributed in the sprayed droplets and can form any possible type of associate upon desolvation of the droplets. The patterns of the ESI mass spectra of the drug-heme mixtures recorded in the present work (Figures 2, 3, and 4) show, that there is a distinct selectivity in complex formation which must proceed in the initial solution. In particular, the association with the heme dimer is characteristic of quinine and is not observed for artemisinin derivatives. Among the artemisinin derivatives that have slight structural differences, only dihydroartemisinin forms a complex with heme with inclusion of a sodium atom. Since dihydroartemisinin is the active metabolite of artemisinin derivatives [1, 30], it can be suggested that interaction of dihydroartemisinin with heme in vivo may involve association with $\mathrm{Na}^{+}$, which is present in all subcellular liquids. This feature is to be accounted for in the development of models of molecular mechanisms of action of artemisinin-type antimalarial agents.

\section{Estimation of the Relative Binding Strength of Drug-Heme Complexes by Low-Energy Collision-Induced Dissociation}

The relative binding strength between the drugs and heme in the noncovalent complexes was assessed using low-energy CID. First, metastable decomposition spectra of the drug-heme complexes (not shown) were obtained, which revealed no elimination of the drug and indicated that the drug-heme complexes are stable in the gas phase. Subsequently, CID spectra were obtained at low and high collision energy using He and $\mathrm{Xe}$, respectively, as collision gas. The reproducibility of the CID conditions was evaluated by monitoring the decomposition of the weakly bound associate of heme with the solvent molecule methanol, i.e., $[\mathrm{FP}: \mathrm{MeOH}]^{+}$ $(m / z$ 648):

$$
[\mathrm{FP} ; \mathrm{MeOH}]^{+} \rightarrow \mathrm{FP}^{+} \mathrm{MeOH}
$$

The RA of the $\mathrm{FP}^{+}$product ion in the CID mass spectra obtained at low collision energy $\left(\mathrm{E}_{\mathrm{com}}=1.8 \mathrm{eV}\right)$ with He was about $42 \pm 2 \%(N=20)$ of that of the precursor [FP:MeOH] ${ }^{+}$selected from any of the studied systems (Figure 5c). Taking into account that the cone voltage affects the signal intensity of the $[\mathrm{FP}: \mathrm{MeOH}]^{+}$ complex, it was also confirmed that it does not decay spontaneously via Pathway 1 . All studied $[\mathrm{M}: \mathrm{FP}]^{+}$ complexes (where $\mathrm{M}$ denotes the drug molecule) decomposed under CID through the neutral loss of the noncovalently bound drug molecule:
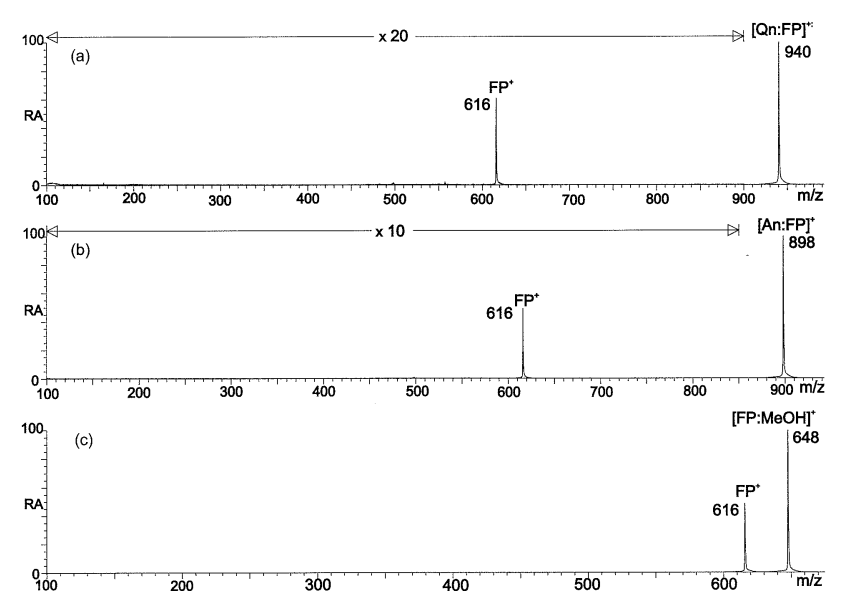

Figure 5. Low-energy CID spectra of heme complexes with (a) quinine, (b) artemisinin, and (c) of the non-specific associate of heme with methanol. The spectra were obtained with helium as collision gas at an $\mathrm{E}_{\text {lab }}$ of $400 \mathrm{eV}$.

$$
[\mathrm{M}: \mathrm{FP}]^{+} \rightarrow \mathrm{FP}^{+}+\mathrm{M}
$$

The CID efficiency of the drug-heme complexes did not depend on the source of a particular complex in that it was practically the same for $[\mathrm{M}: \mathrm{FP}]^{+}$ions selected in experiments using mixtures with different number and ratio of the interacting components. As could be expected, a dependence on the collision gas was observed. Figures 5 and 6 illustrate the CID mass spectra obtained with $\mathrm{He}$ and $\mathrm{Xe}$, respectively, as collision gas, for two complexes $[\mathrm{Qn}: \mathrm{FP}]^{+}$(a) and $[\mathrm{An}: \mathrm{FP}]^{+}$(b). It can be noted that the $\mathrm{RA}$ of the product ion $\mathrm{FP}^{+}$is higher for high-energy CID with Xe. Furthermore, product ions at lower $m / z$ such as the ion at $m / z 557$, due to fragmentation of $\mathrm{FP}^{+}$, were generated, as could be expected for high-energy CID. In contrast, low-energy CID with He only resulted in the elimination of the noncovalently bound drug molecule and was, therefore, selected in further experiments aimed at determining the relative binding strengths of the drug-heme complexes.

Taking into account that DHAn is the only compound among the evaluated artimisinin-type drugs which forms two types of complexes with heme (Table 2), the CID mass spectrum (Figure 7) was obtained for

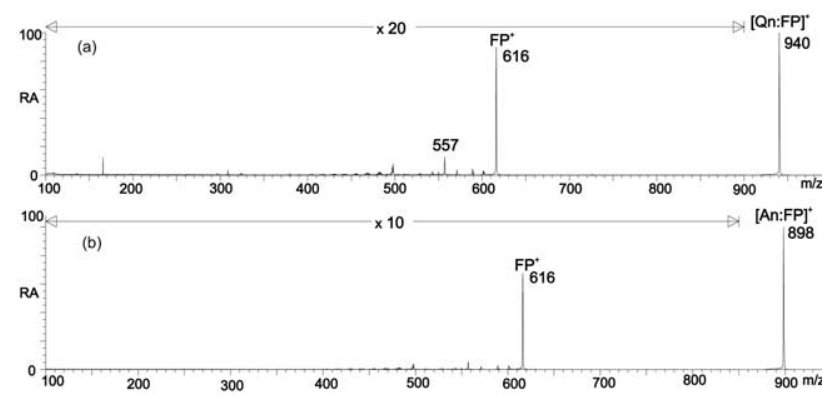

Figure 6. High-energy CID spectra of heme complexes with (a) quinine and (b) artemisinin. The spectra were obtained with xenon as collision gas at an $\mathrm{E}_{\text {lab }}$ of $400 \mathrm{eV}$. 


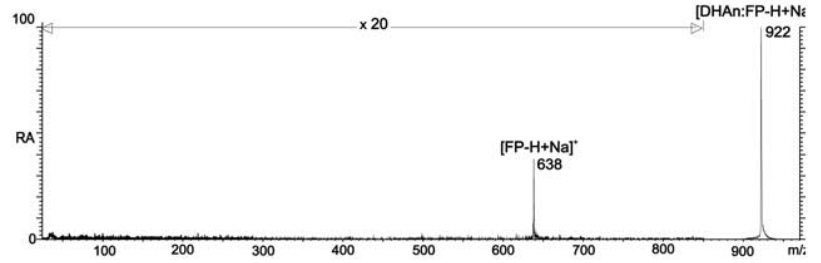

Figure 7. Low-energy CID spectra of the $\mathrm{Na}^{+}$-containing heme complex with dihydroartemisinin. The spectrum was obtained with helium as collision gas at an $\mathrm{E}_{\mathrm{lab}}$ of $400 \mathrm{eV}$.

[DHAn:FP $-\mathrm{H}+\mathrm{Na}^{+}$( $m / z$ 922) complex, for which the following decomposition was observed:

$$
\begin{aligned}
{[\text { DHAn:FP }} & -\mathrm{H}+\mathrm{Na}]^{+} \\
& \rightarrow[\mathrm{FP}-\mathrm{H}+\mathrm{Na}]^{+}+\text {DHAn }
\end{aligned}
$$

This low-energy CID experiment thus reveals that the Na-containing dihydroartemisinin:heme complex $(m / z$ 922) results in Na-containing heme $(m / z$ 638), in which the $\mathrm{Na}$ atom can be located in one of the two ethylcarboxyl side chains. It is suggested that during complex formation the $\mathrm{Na}$ atom is transferred from the sodiated dihydroartemisinin molecule (detected at $\mathrm{m} / \mathrm{z}$ 307; Table 1), most likely from the 12-hydroxyl group which is part of a hemiketal function and has acidic properties.

The relative abundances of the product ions $\mathrm{FP}^{+}$or $[\mathrm{FP}-\mathrm{H}+\mathrm{Na}]^{+}$, obtained in low-energy CID of different drug-heme complexes, averaged using the data of three to eight independent measurements, are summarized in Table 3.

The analysis of these data reveals a nearly two-fold lower CID efficiency of the $[\mathrm{Qn}: \mathrm{FP}]^{+}$complex in comparison with that of all other complexes $[\mathrm{M}: \mathrm{FP}]^{+}$, indicating a higher stability of the quinine-heme complex. The relative stabilities of all complexes of heme with the artemisinin-type drugs as determined by CID of the $\left[\mathrm{M}: \mathrm{FP}^{+}\right.$complexes are comparable within the experimental error. It is interesting to note that the stability of the [DHAn:FP $-\mathrm{H}+\mathrm{Na}]^{+}$complex is as high as that of the $[\mathrm{Qn}: \mathrm{FP}]^{+}$complex. The increase of the stability of the drug-heme complex containing $\mathrm{a} \mathrm{Na}^{+}$ion can be

Table 3. Relative abundances and relative standard deviations of product ions obtained in low-energy CID (He) of different

\begin{tabular}{|c|c|}
\hline \multirow[b]{2}{*}{ Complex } & RA of product ion, $\%$ \\
\hline & $\mathrm{FP}^{+} \mathrm{m} / \mathrm{z} 616$ \\
\hline$[\mathrm{On}: \mathrm{FP}]^{+}$ & $2.78 \pm 0.22(\mathrm{~N}=5)$ \\
\hline$[\mathrm{An}: \mathrm{FP}]^{+}$ & $5.6 \pm 0.25(\mathrm{~N}=8)$ \\
\hline$[\mathrm{DHAn:FP}]^{+}$ & $5.86 \pm 0.35(\mathrm{~N}=4)$ \\
\hline$[\alpha \mathrm{Am}: \mathrm{FP}]^{+}$ & $5.13 \pm 0.26(\mathrm{~N}=4)$ \\
\hline$[\beta \mathrm{Am}: \mathrm{FP}]^{+}$ & $4.98 \pm 0.44(\mathrm{~N}=4)$ \\
\hline$[\mathrm{AE}: \mathrm{FP}]^{+}$ & $5.33 \pm 0.31(\mathrm{~N}=3)$ \\
\hline & {$[\mathrm{FP}-\mathrm{H}+\mathrm{Na}]^{+} \mathrm{m} / \mathrm{z} 638$} \\
\hline$[\mathrm{DHAn}: \mathrm{FP}-\mathrm{H}+\mathrm{Na}]^{+}$ & $1.93 \pm 0.16(\mathrm{~N}=3)$ \\
\hline
\end{tabular}
drug-heme complexes explained by an increased electrostatic interaction, which has been reported as the main component of noncovalent interaction in the complex [20]. Since DHAn is known to be the active metabolite of artemisinin derivatives, it can be suggested that the DHAnheme interaction in vivo may also involve sodium ions. We have stated in the introduction that formation of a relatively stable noncovalent complex between heme and drugs of the artemisinin-type can be regarded as the first step in covalent interaction between the ligand and heme, since $\mathrm{Fe}^{2+}$ is required for activation of artemisinin derivatives, i.e., generation of alkyl radicals. In this respect, it has been found experimentally in in vitro experiments by Messori et al. [19] that the covalent association is slightly faster for dihydroartemisinin compared with other artemisinin derivatives, a feature that could be explained by the initial formation of a more stable noncovalent heme-dihydroartemisinin complex.

The comparison of the CID behavior of all drugheme complexes with that of $[\mathrm{FP}: \mathrm{MeOH}]^{+}$(Figure 5c) shows, that the binding between the components of this non-specific associate of heme with the solvent molecule methanol is about 10 times weaker than between the components of specific drug-heme complexes. In contrast to the ESI-MS data obtained for the $\alpha$ - and $\beta$-isomers of artemether, indicating that the $\alpha$-isomer is about 1.5 times more efficient than the $\beta$-isomer in binding with heme, the CID data reveal that their binding strengths are comparable within the experimental error. The reason for this discrepancy may be due to the fact that solvent effects play a role in noncovalent complex formation in the bulk solution.

\section{Conclusions}

In this study, we have demonstrated, using ESI-MS and ESI-MS/CID/MS, that stable noncovalent complexes can be formed between Fe(III)-heme and antimalarial agents, i.e., quinine, artemisinin, and the artemisinin derivatives, dihydroartemisinin, $\alpha$ - and $\beta$-artemether, and $\beta$-arteether. Differences in the binding behavior of the examined drugs with Fe(III)-heme and the stability of the drug-heme complexes were demonstrated. The results show that all tested antimalarial agents form a drug-heme complex with a 1:1 stoichiometry but that quinine also results in a second complex with the heme dimer or $\beta$-hematin. ESI-MS experiments performed on mixtures of the various antimalarial agents with heme indicate that quinine binds preferentially to $\mathrm{Fe}(\mathrm{III})$ heme, while ESI-MS/CID/MS shows that the quinineheme complex is nearly two times more stable than the complexes formed between heme and artemisinin and its derivatives. Moreover, it was found that dihydroartemisinin, the active metabolite of the artemisinin-type drugs in vivo, results in a $\mathrm{Na}^{+}$-containing heme-drug complex, which is as stable as the heme-quinine complex. The efficiency of drug-heme binding of artemisinin derivatives is generally lower and the decomposi- 
tion under CID higher compared with quinine, but these parameters are within the same order of magnitude. These results suggest that the efficiency of antimalarial agents of the artemisinin-type to form noncovalent complexes with Fe(III)-heme is comparable with that of the traditional antimalarial agent quinine. While the noncovalent complexes between Fe(III)-heme and artemisinin, artemether or arteether, were stable in electrospray ionization conditions, electrochemical reactions were observed for the dihydroartemisinin-heme complex.

Our study illustrates that electrospray ionization mass spectrometry and collision-induced dissociation tandem mass spectrometry are suitable tools to probe noncovalent interactions between heme and antimalarial agents. The results obtained provide insights into the underlying molecular modes of action of the traditional antimalarial agent quinine and of the antimalarials of the artemisinin-type which are currently used to treat severe or multidrug-resistant malaria.

\section{Acknowledgments}

This work has been supported by the Special Research Fund of the University of Antwerp through a BOF concerted action (grant no. 99/3/34) and a visiting postdoctoral fellowship to VP. The authors are grateful to Dr. F. H. Janssen (Dafra Pharma, OudTurnhout, Belgium) for the generous gift of artimisinin derivatives and for stimulating discussions.

\section{References}

1. Jansen, F. H. Artesunate and Artemether. Towards the Eradication of Malaria? Dafra Pharma Ltd: Oud-Turnhout, 2002; 1.

2. Guterl, F. Battle Against the Bugs. Sci. Technol. 2002, 14, 51-53.

3. Warthurt, D. C.; Craig, J. C.; Adagu, I. S. Lysosomes and Drug Resistance in Malaria. Lancet 2002, 360, 1527-1529.

4. Padmanaban, G.; Rangarajan, P. N. Heme Metabolism of Plasmodium is a Major Antimalarial Target. Biochem. Biophys. Res. Commun. 2000, 268, 665-668.

5. Slater, A. F. Chloroquine: Mechanism of Drug Action and Resistance in Plasmodium Falciparum. Pharmacol. Ther. 1993, 57, 203-235.

6. Sullivan, D. J.; Matile, H.; Ridley, R. G.; Goldberg, D. E. A Common Mechanism for Blockade of Heme Polymerization by Antimalarial Quinolines. J. Biol. Chem. 1998, 273, 3110331107.

7. Loria, P.; Miller, S.; Foley, M.; Tilley, L. Inhibition of the Peroxidative Degradation of Haem as the Basis of Action of Chloroquine and Other Quinoline Antimalarials. Biochem. J. 1999, 339, 363-370.

8. Pagola, S.; Stephens, P. W.; Bohle, D. S.; Kosar, A. D.; Madesn, S. K. The Structure of Malaria Pigment $\beta$-Haematin. Nature 2000, 404, 307-310.

9. Mann, R. D. Modern Drug Use: An Enquiry on Historical Principles; MTP Press, Ltd.: Lancaster, UK, 1984; p 268.

10. Jansen, F. H.; Yin, Z. Who Discovered Artemisinin? In Artesunate and Artemether. Towards the Eradication of Malaria? Jansen, F. H., Ed.; Dafra Pharma Ltd: Oud-Turnhout, Belgium, 2002; p 25.

11. Benoit-Vical, F.; Robert, A.; Meunier, B. In Vivo and in Vitro Potentiation of Artemisinin and Synthetic Endoperoxide Antimalarial Drugs by Metalloporphyrins. Antimicrob. Agents Chemother. 2000, 44, 2836-2841.
12. Olliaro, P. L.; Haynes, R. K.; Meunier, B.; Yuthavong, Y. Possible Modes of Action of the Artemisinin-Type Compounds. Trends Parasitol. 2001, 17, 122-126.

13. Meshnick, S. R.; Taylor, T. E.; Kamchonwongpaisan, S. Artemisinin and the Antimalarial Endoperoxides: From Herbal Remedy to Targeted Chemotherapy. Microbiol. Rev. 1996, 60, 301-315.

14. RobertA.CoppelY.MeunierB. Alkylation of Heme by the Antimalarial Drug Artemisinin. J. Chem. Soc. Chem. Commun. 2002, 414-415.

15. WangD.-Y.WuY.-L.WuY.LiangJ.LiY. Further Evidence for Participation of Primary Carbon-Centered Free Radicals in the Antimalarial Action of the Qinghaosu (Artemisinin) Series of Compounds. J. Chem. Soc. Perkin Trans. 2002, 65-609.

16. Kannan, R.; Sahal, D.; Chauhan, V. S. Heme-Artemisinin Adducts are Crucial Mediators of the Ability of Artemisinin to Inhibit Heme Polymerization. Chem. Biol. 2002, 9, 321-332.

17. Eckstein-Ludwig, U.; Webb, R. J.; van Goethem, I. D. A.; East, J. M.; Lee, A. G.; Kimura, M.; O’Neill, P. M.; Bray, P. G.; Ward, S. A.; Krishna, S. Artemisinins Target the SERCA of Plasmodium falciparum. Nature 2003, 424, 957-961.

18. Paitayatat, S.; Tarnchompoo, B.; Thebtaranonth, Y.; Yuthavong, Y. Correlation of Antimalarial Activity of Artemisinin Derivatives with Binding Affinity with Ferroprotoporphyrin IX. J. Med. Chem. 1997, 40, 633-638.

19. Messori, L.; Piccioli, F.; Eitler, B.; Bergonzi, M. C.; Bilia, A. R.; Vincieri, F. F. Spectrophotometric and ESI-MS/HPLC Studies Reveal a Common Mechanism for the Reaction of Various Artemisinin Analogues with Hemin. Bioorg. Med. Chem. 2003, 13, 4055-4057.

20. Cheng, F.; Shen, J.; Luo, X.; Zhu, W.; Gu, J.; Ji, R.; Jiang, H.; Chen, K. Molecular Docking and 3-D-QSAR Studies on the Possible Antimalarial Mechanism of Artemisinin Analogues. Bioorg. Med. Chem. 2002, 10, 2883-2891.

21. Cole, R. B. Electrospray Ionization Mass Spectrometry. Fundamentals, Instrumentation and Applications; John Wiley and Sons: New York, 1997; p 578.

22. Przybylski, M.; Glocker, M. O. Electrospray Mass Spectrometry of Biomacromolecular Complexes with Noncovalent Interactions-New Analytical Perspectives for Supramolecular Chemistry and Molecular Recognition Processes. Angew. Chem. Int. Ed. 1996, 108, 878-899.

23. Veenstra, T. D. Electrospray Ionization Mass Spectrometry in the Study of Biomolecular Noncovalent Interactions. Biophys. Chem. 1999, 79, 63-79.

24. Skribanek, Z.; Balaspiri, L.; Mák, M. Interaction Between Synthetic Amyloid- $\beta$-Peptide (1-40) and its Aggregation Inhibitors Studied by Electrospray Ionization Mass Spectrometry. J. Mass Spectrom. 2001, 36, 1226-1229.

25. Loo, J. A. Studying Noncovalent Protein Complexes by Electrospray Ionization Mass Spectrometry. Mass Spectrom. Rev. 1997, 16, 1-23.

26. Li, Y.-T.; Hsieh, Y.-L.; Henion, J. D. Studies on Heme Binding in Myoglobin, Hemoglobin, and Cytochromec by Ion Spray Mass Spectrometry. J. Am. Soc. Mass Spectrom. 1993, 4, 631-637.

27. Wright, A. D.; Wang, H.; Gurrath, M.; König, G. M.; Kocak, G.; Neumann, G.; Loria, P.; Foley, M.; Tilley, L. Inhibition of Heme Detoxification Processes Underlies the Antimalarial Activity of Terpene Isonitrile Compounds from Marine Sponges. J. Med. Chem. 2001, 44, 873-885.

28. Jonckers, T. H. M.; Van Miert, S.; Cimanga, K.; Bailly, C.; Colson, P.; De Pauw-Gillet, M.-C.; Van den Heuvel, H.; Claeys, M.; Lemière, F.; Esmans, E. L.; Rozenski, J.; Quirijnen, L.; Maes, L.; Dommisse, R.; Lemière, G. L. F.; Vlietinck, A.; Pieters, L. Synthesis, Cytotoxicity, and Antiplasmodial and Antitrypanosomal Activity of New Neocryptolepine Derivatives. J. Med. Chem. 2002, 45, 3497-3508. 
29. Cech, N. B.; Enke, C. G. Practical Implications of Some Recent Studies in Electrospray Ionization Fundamentals. Mass Spectrom. Rev. 2001, 20, 362-387.

30. Grace, J. M.; Aguilar, A. J.; Trotman, K. M.; Brewer, T. G. Metabolism of $\beta$-Arteether to Dihydroginghaosu by Human Liver Microsomes and Recombinant Cytochrome P450. Drug Metab. Dispos. 1998, 26, 313-317.

31. Aplin, R. T.; Robinson, C. V.; Schofield, C. J.; Westwood, N. J. Does the Observation of Noncovalent Complexes between
Biomolecules by Electrospray Ionization Mass Spectrometry Necessarily Reflect Specific Solution Interactions? J. Chem. Soc. Chem. Commun. 1994, 2415-2417.

32. Kebarle, P. A Brief Overview of the Present Status of the Mechanisms Involved in Electrospray Mass Spectrometry. J. Mass Spectrom. 2000, 35, 804-817.

33. Loo, J. A. Electrospray Ionization Mass Spectrometry: A Technology for Studying Noncovalent Macromolecular Complexes. Int. J. Mass Spectrom. 2000, 200, 175-186. 